\title{
STRUCTURE AND STABILITY OF FINITE DIMENSIONAL APPROXIMATIONS FOR FUNCTIONAL DIFFERENTIAL EQUATIONS*
}

\author{
DIETMAR SALAMON $\dagger$
}

\begin{abstract}
This paper deals with the structural and stability properties of the averaging approximation scheme for linear retarded functional differential equations. Both in the discrete- and in the continuous-time case the structure of the approximating systems is shown to be analogous to the structure of the underlying retarded equation. Moreover, it is shown that the approximating systems are exponentially stable in a uniform sense if the original system is asymptotically stable.
\end{abstract}

AMS (MOS) subject classifications. 34K99, 65L07 stability

Key words. functional differential equations, averaging approximation, structural operators, uniform

1. Introduction. The object of this paper is to present some new results on the averaging approximation scheme for linear retarded functional differential equations (RFDE).

The averaging approximation scheme has been invented and studied by several Soviet authors in the early sixties (see e.g. Repin [18]; further references and a detailed review can be found in Banks-Burns [2]). A general convergence proof, a stability analysis and applications to optimal control problems have been presented for the first time by Banks-Burns [1], [2]. Related discrete-time approximations have been considered by Delfour [6], Reber [17], Rosen [19]. Recently, Gibson [9] has used the averaging scheme for approximating the solution of the algebraic Riccati equation associated with a retarded system. However, there remained one open problem in the convergence proof in [9] which has not yet been resolved. This is the question whether the approximating systems are uniformly exponentially stable for sufficiently large $N$ if the underlying RFDE is stable. In [9] this has been stated as a conjecture without proof. We show in $\S 4.2$ that this conjecture is in fact correct.

Another motivation for the present work comes from some recent developments in the theory of retarded systems in the product space framework. One of these is the introduction of so-called structural operators for the state space description of RFDE's which have made the linear theory much more elegant and efficient (see e.g. BernierManitius [3], Manitius [14], Delfour-Manitius [7]). They have led to a number of new results in the control theory of RFDE's, namely on problems like completeness of eigenfunctions, controllability, observability, and the linear quadratic optimal control problem. Another important development was an interpretation of the adjoint semigroup in terms of the underlying RFDE. Extensions to neutral systems and further references can be found in Salamon [20].

The problem has not yet been considered whether analogous results can be developed for finite dimensional approximation of RFDE's, in particular the averaging approximation scheme. In this paper we fill this gap. It is shown that the approximating systems satisfy analogous duality relations as the RFDE and certain structural matrices are introduced which play an analogous role for the approximating systems as the

\footnotetext{
* Received by the editors October 18, 1983, and in revised form May 28, 1984. This research was sponsored by the U.S. Army under contract DAAG29-80-C-0041 and by the National Science Foundation under grant MCS-8210950. The material is based upon work supported by the Forschungsschwerpunkt "Dynamische Systeme", University of Bremen, West Germany.

$\dagger$ Mathematics Research Center, University of Wisconsin-Madison, Madison, Wisconsin 53706.
} 
structural operators do for the RFDE. Moreover, it is shown that these matrices actually converge to the corresponding structural operators. These results have several important consequences. For example, they lead to a uniform convergence result for the resolvent operators and they are crucial for the proofs of the stability results in $\S 4.2$.

In the preliminary $\S 2$ we give a brief overview over some recent results in the theory of linear retarded systems in the product space framework and describe the averaging approximation scheme. Section 3 is devoted to the study of the structure of the approximating systems which is shown to be analogous to the structure of the underlying RFDE under several aspects. A number of convergence proofs is then given in $\S 4.1$ and two stability results are proved in $\S 4.2$. In the appendix $(\S 6)$ we prove two functional analytic results which are frequently needed in $\$ 4$. In particular, we give a quantitative estimate for the equivalence of $L^{P}$-stability and exponential stability for strongly continuous semigroups.

2. Linear retarded systems and averaging approximation.

2.1. Linear retarded systems. We consider the linear retarded functional differential equation

$$
\dot{x}(t)=L x_{t}, \quad t \geqq 0,
$$

where $x(t) \in \mathbb{R}^{n}$ and $x_{t}$ is defined by $x_{t}(\tau)=x(t+\tau),-h \leqq \tau \leqq 0, h>0$. Correspondingly $L$ is a bounded linear functional from $\mathscr{C}=\mathscr{C}\left[-h, 0 ; \mathbb{R}^{n}\right]$ into $\mathbb{R}^{n}$ given by

$$
L \phi=\int_{-h}^{0} d \eta(\tau) \phi(\tau), \quad \phi \in \mathscr{C},
$$

where $\eta(\tau)$ is an $n \times n$-matrix valued function of bounded variation. Without loss of generality we can assume that $\eta$ is normalized which means that $\eta(\tau)=0$ for $\tau \geqq$ $0, \eta(\tau)=\eta(-h)$ for $\tau \leqq-h$, and $\eta(\tau)$ is left continuous for $-h<\tau<0$. At some places we will assume that $L$ is given by

$$
L \phi=\sum_{j=0}^{q} A_{j} \phi\left(-h_{j}\right)+\int_{-h}^{0} A_{01}(\tau) \phi(\tau) d \tau, \quad \phi \in \mathscr{C},
$$

where $0=h_{0}<\cdots<h_{q}=h$ and $A_{j} \in \mathbb{R}^{n \times n}, j=0, \cdots, q$ as well as $A_{01}(\cdot) \in$ $L^{2}\left[-h, 0 ; \mathbb{R}^{n \times n}\right]$. In this case $\eta: \mathbb{R} \rightarrow \mathbb{R}^{n \times n}$ is clearly given by

$$
\eta(\tau)=-A_{0} \chi_{(-\infty, 0)}(\tau)-\sum_{j=1}^{q} A_{j} \chi_{\left(-\infty,-h_{j}\right]}(\tau)-\int_{\tau}^{0} A_{01}(\sigma) d \sigma, \quad \tau \in \mathbb{R},
$$

where $\chi_{I}$ denotes the characteristic function of the interval $I$.

It is well known that (2.1) admits a unique solution $x(\cdot) \in$ $L_{\text {loc }}^{2}\left[-h, \infty ; \mathbb{R}^{n}\right] \cap W_{\text {loc }}^{1,2}\left[0, \infty ; \mathbb{R}^{n}\right]$ for every initial condition of the form

$$
x(0)=\phi^{0}, \quad x(\tau)=\phi^{1}(\tau), \quad-h \leqq \tau<0,
$$

where $\phi .=\left(\phi^{0}, \phi^{1}\right) \in \mathbb{R}^{n} \times L^{2}\left[-h, 0 ; \mathbb{R}^{n}\right]=: M^{2}$. This solution depends continuously on $\phi \in M^{2}$. The fundamental solution of (2.1) will be denoted by $X(t) \in \mathbb{R}^{n \times n}, t \geqq-h$, and corresponds to the initial condition $X(0)=I, X(\tau)=0,-h \leqq \tau<0$. It can also be characterized by the Volterra integral equation

$$
X(t)=I-\int_{0}^{t} \eta(s-t) X(s) d s
$$

and its Laplace transform is given by $\Delta(\lambda)^{-1}$ where $\Delta(\lambda)=\lambda I-L\left(e^{\lambda \cdot}\right), \lambda \in \mathbb{C}$, is the characteristic matrix of (2.1). 
Proofs of these facts can be found e.g. in Hale [10] or Delfour-Manitius [7].

2.2. Semigroups and structural operators. In the theory of RFDE's, as well as other types of integral and functional differential equations, there are essentially two ways of introducing the state of the system which are actually dual to each other. The state of system (2.1) in the "classical" sense is the pair $z(t)=\left(x(t), x_{t}\right) \in M^{2}$ which completely describes the past history of the solution. Its evolution determines the strongly continuous semigroup $S(t)$ of bounded linear operators on $M^{2}$ defined by

$$
S(t) \phi=\left(x(t), x_{t}\right) \in M^{2}, \quad \phi \in M^{2}, \quad t \geqq 0,
$$

where $x(t), t \geqq-h$, is the unique solution of (2.1) and (2.3). The infinitesimal generator of $S(t)$ is given by

$$
\begin{aligned}
& \operatorname{dom} A=\left\{\phi \in M^{2} \mid \phi^{1} \in W^{1,2}, \phi^{0}=\phi^{1}(0)\right\}, \\
& A \phi=\left(L \phi^{1}, \dot{\phi}^{1}\right),
\end{aligned}
$$

where $W^{1,2}$ denotes the Sobolev space $W^{1,2}\left[-h, 0, \mathbb{R}^{n}\right]$. In an analogous way we may introduce the semigroup $S_{T}(t) \in \mathscr{L}\left(M^{2}\right), t \geqq 0$, with infinitesimal generator $A_{T}$ corresponding to the transposed RFDE

$$
\dot{x}(t)=L^{T} x_{t}, \quad t \geqq 0 .
$$

The duality relation between (2.1) and (2.4) can be described by means of an alternative (dual) state concept which is due to Miller [15]. It can be motivated from the fact that the solution of the RFDE $(2.1)(t>0)$ can be derived from the initial function $(t \leqq 0)$ in two steps. First convert the initial function $\phi^{1}$ into a forcing term of suitable length which determines the future behaviour of the solution. Secondly determine the solution which corresponds to this forcing term. The dual state concept is obtained by regarding this forcing term as the initial state of the system rather than the solution segment. To be more precise, we rewrite (2.1) as

$$
\dot{x}(t)=\int_{-t}^{0} d \eta(\tau) x(t+\tau)+f^{1}(-t), \quad x(0)=f^{0},
$$

where the pair $f=\left(f^{0}, f^{1}\right) \in M^{2}$ is given by

$$
f^{0}=\phi^{0}, f^{1}(\sigma)=\int_{-h}^{\sigma} d \eta(\tau) \phi^{1}(\tau-\sigma), \quad-h \leqq \sigma \leqq 0 .
$$

Now the initial state of $(2.5)$ is given by $f \in M^{2}$. Correspondingly the state at time $t \geqq 0$ is the pair $w(t)=\left(x(t), x^{t}\right) \in M^{2}$ where $x^{t} \in L^{2}\left[-h, 0 ; \mathbb{R}^{n}\right]$ denotes the forcing term of the shifted equation (2.5) and is given by

$$
x^{t}(\sigma)=\int_{\sigma-t}^{\sigma} d \eta(\tau) x(t+\tau-\sigma)+f^{1}(\sigma-t), \quad-h \leqq \sigma \leqq 0 .
$$

The evolution of this state $\left(x(t), x^{t}\right) \in M^{2}$ is described by the semigroup $S_{T}^{*}(t)$ (see e.g. Bernier-Manitius [3] or Salamon [20]).

Summarizing this situation, we have to deal with the following four semigroups:

$$
\begin{array}{ll}
S(t) & S_{T}(t) \\
S_{T}^{*}(t) & S^{*}(t) .
\end{array}
$$

The semigroups on the left correspond to the RFDE (2.1) and those on the right to the transposed RFDE (2.4). On each side the upper semigroup describes the respective 
equation within the "classical" state concept (solution segments) and the one below within the dual state concept (forcing terms). The diagonal relations are actually given by functional analytic duality.

The relation between the two state concepts can be described by means of so-called structural operators. These have been introduced by Bernier-Manitius [3], Manitius [14], Delfour-Manitius [7] and have turned out to be a very elegant and efficient concept in the control theory of RFDE's. The operator $F \in \mathscr{L}\left(M^{2}\right)$ maps every $\phi \in M^{2}$ into the corresponding initial state

$$
F \phi=f \in M^{2}
$$

of (2.5) which is given by (2.6). The operator $G \in \mathscr{L}\left(M^{2}\right)$ maps every forcing term $f \in M^{2}$ into the corresponding solution segment

$$
G f=\left(x(h), x_{h}\right) \in M^{2}
$$

of (2.5) at time $h$. Thus $G f$ can be explicitly described as

$$
\begin{aligned}
& {[G f]^{0}=[G f]^{1}(0),} \\
& {[G f]^{1}(\tau)=X(h+\tau) f^{0}+\int_{0}^{h} X(h+\tau-s) f^{1}(-s) d s, \quad-h \leqq \tau \leqq 0 .}
\end{aligned}
$$

Obviously, $G$ is bijective as an operator from $M^{2}$ into $\operatorname{dom} A$ and its inverse is given by

$$
\begin{aligned}
& {\left[G^{-1} \phi\right]^{0}=\phi^{1}(-h),} \\
& {\left[G^{-1} \phi\right]^{1}(\sigma)=\dot{\phi}^{1}(-\sigma-h)-\int_{\sigma}^{0} d \eta(\tau) \phi^{1}(\tau-\sigma-h), \quad-h \leqq \sigma \leqq 0,}
\end{aligned}
$$

for $\phi \in \operatorname{dom} A$. A remarkable fact is that the adjoint operators $F^{*}$ and $G^{*}$ play the same role for the transposed equation (2.4) as the operators $F$ and $G$ do for the original equation (2.1). Moreover, the following result has been proved by Manitius [14] and Delfour-Manitius [7].

THEOREM 2.1.

(i) $S(h)=G F, S_{T}^{*}(h)=F G$.

(ii) $F S(t)=S_{T}^{*}(t) F, S(t) G=G S_{T}^{*}(t), t \geqq 0$.

(iii) If $\phi \in \operatorname{dom} A$, then $F \phi \in \operatorname{dom} A_{T}^{*}$ and $A_{T}^{*} F \phi=F A \phi$.

(iv) If $f \in \operatorname{dom} A_{T}^{*}$, then $G A_{T}^{*} f=A G f$.

We close this section with a concrete representation of the resolvent operator. For this sake we introduce for any $\lambda \in \mathbb{C}$ the operators $E_{\lambda}: \mathbb{C}^{n} \rightarrow M^{2}$ and $T_{\lambda}: M^{2} \rightarrow M^{2}$ by defining

$$
\begin{array}{ll}
{\left[E_{\lambda} x\right]^{0}=x,} & {\left[E_{\lambda} x\right]^{1}(\tau)=e^{\lambda \tau} x, \quad x \in \mathbb{C}^{n},} \\
{\left[T_{\lambda} \phi\right]^{0}=0,} & {\left[T_{\lambda} \phi\right]^{1}(\tau)=\int_{\tau}^{0} e^{\lambda(\tau-\sigma)} \phi^{1}(\sigma) d \sigma, \quad \phi \in M^{2} .}
\end{array}
$$

Then the following result has been proved in Manitius [13] and Delfour-Manitius [7].

Proposition 2.2. Let $\operatorname{det} \Delta(\lambda) \neq 0$. Then

$$
\begin{aligned}
& (\lambda I-A)^{-1}=E_{\lambda} \Delta(\lambda)^{-1} E_{\bar{\lambda}}^{*} F+T_{\lambda}, \\
& \left(\lambda I-A_{T}^{*}\right)^{-1}=F E_{\lambda} \Delta(\lambda)^{-1} E_{\bar{\lambda}}^{*}+T_{\bar{\lambda}}^{*} .
\end{aligned}
$$

2.3. Averaging approximation. In this section we briefly describe an approximation scheme for RFDE's which has been studied by Repin [18], Banks-Burns [1], [2], 
Gibson [9] and many others. To this end we introduce for every $N \in \mathbb{N}$ the linear subspace $X^{N} \subset M^{2}$ defined by

$$
X^{N}=\left\{\phi \in M^{2} \mid \phi^{1}(\tau)=z_{j} \in \mathbb{R}^{n},-\frac{j}{n} h \leqq \tau<-\frac{j-1}{N} h, \quad j=1, \cdots, N\right\}
$$

and denote the corresponding orthogonal projection by $p^{N}: M^{2} \rightarrow X^{N}$. This subspace can be identified with $\mathbb{R}^{n(N+1)}$ by means of the embedding $\iota^{N}: \mathbb{R}^{n(N+1)} \rightarrow M^{2}$ which associates with every $z=\left(z_{0}^{T}, \cdots, z_{N}^{T}\right)^{T} \in \mathbb{R}^{n(N+1)}$ the pair

$$
\begin{aligned}
& {\left[\iota^{N} z\right]^{0}=z_{0},} \\
& {\left[\iota^{N} z\right]^{1}(\tau)=z_{j}, \quad-\frac{j}{N} h \leqq \tau<-\frac{j-1}{N} h, \quad j=1, \cdots, N .}
\end{aligned}
$$

On $\mathbb{R}^{n(N+1)}$ we will always consider the induced inner product

$$
\langle z, w\rangle_{N}=z^{T} Q^{N} w, \quad z, w \in \mathbb{R}^{n(N+1)},
$$

where

$$
Q^{N}=\left[\begin{array}{cccc}
I & 0 & \cdots & 0 \\
0 & \frac{h}{N} I & \ddots & \vdots \\
\vdots & \ddots & \ddots & 0 \\
0 & \cdots & 0 & \frac{h}{N} I
\end{array}\right]
$$

The corresponding vector and matrix norms will be denoted by $\|\cdot\|_{N}$. The adjoint operator $\pi^{N}=\left(\iota^{N}\right)^{*}: M^{2} \rightarrow \mathbb{R}^{n(N+1)}$ is then given by

$$
\left[\pi^{N} \phi\right]_{0}=\phi^{0}, \quad\left[\pi^{N} \phi\right]_{j}=\frac{N}{h} \int_{-j h / N}^{-(j-1) h / N} \phi^{1}(\tau) d \tau, \quad j=1, \cdots, N .
$$

Obviously, the operators $\iota^{N}$ and $\pi^{N}$ satisfy

$$
\pi^{N} \iota^{N}=\mathrm{id}, \quad \iota^{N} \pi^{N}=p^{N} .
$$

On $\mathbb{R}^{n(N+1)}$ we consider the differential equation

$$
\dot{z}^{N}(t)=A^{N} z^{N}(t), \quad t \geqq 0,
$$

where

$$
A^{N}=\left(Q^{N}\right)^{-1} H^{N}, \quad H^{N}=\left[\begin{array}{cccc}
A_{0}^{N} & A_{1}^{N} & \cdots & A_{N}^{N} \\
I & -I & & \\
& \ddots & \ddots & \\
& & I & -I
\end{array}\right] .
$$

and

$$
A_{j}^{N}=\lim _{\tau \uparrow-j h / N}\left[\eta\left(\tau+\frac{h}{N}\right)-\eta(\tau)\right], \quad j=0, \cdots, N .
$$

In an analogous way we define the matrix $A_{T}^{N}=\left(Q^{N}\right)^{-1} H_{T}^{N}$ where the $A_{j}^{N}$ are replaced by $\left(A_{j}^{N}\right)^{T}$ for $j=0,1, \cdots, N$. Then the adjoint matrix $\left(A_{T}^{N}\right)^{*}$ of $A_{T}^{N}$ with respect to 
the inner product $\langle\cdot, \cdot\rangle_{N}$ is given by

$$
\left(A_{T}^{N}\right)^{*}=\left(Q^{N}\right)^{-1}\left(H_{T}^{N}\right)^{T}, \quad\left(H_{T}^{N}\right)^{T}=\left[\begin{array}{rrrr}
A_{0}^{N} & I & & \\
A_{1}^{N} & -I & \ddots & \\
\vdots & & \ddots & I \\
A_{N}^{N} & & & -I
\end{array}\right] .
$$

We also consider the differential equation

$$
\dot{w}^{N}(t)=\left(A_{T}^{N}\right)^{*} w^{N}(t), \quad t \geqq 0,
$$

on $\mathbb{R}^{n(N+1)}$. The following theorem has been proved in Banks-Burns [2] and Gibson [9].

THEOREM 2.3. Let $L: \mathscr{C} \rightarrow \mathbb{R}^{n}$ be given by (2.2). Then the following statements hold.

(i) For every $\phi \in M^{2}$ we have $\phi=\lim _{N \rightarrow \infty} p^{N} \phi$.

(ii) There exist constants $M \geqq 1, \omega \geqq 0$, such that

$$
\left\|e^{A^{N} t}\right\|_{N} \leqq M e^{\omega t}, \quad\left\|e^{\left(A_{T}^{N}\right)^{*} t}\right\|_{N} \leqq M e^{\omega t}
$$

for every $t \geqq 0$ and every $N \in \mathbb{N}$.

(iii) For all $\phi \in M^{2}, f \in M^{2}$

$$
S(t) \phi=\lim _{N \rightarrow \infty} \iota^{N} e^{A^{N} t} \pi^{N} \phi, \quad S_{T}^{*}(t) f=\lim _{N \rightarrow \infty} \iota^{N} e^{\left(A_{T}^{N}\right)^{*} t} \pi^{N} f
$$

and the limits are uniform on every compact interval $[0, T]$.

Full discretization. A fairly general and extensive study of full discretization methods for RFDE's can be found in Reber [17] and Rosen [19]. Since the aim of this work is to explore the special structure of the averaging approximation scheme described above, we content ourselves with the consideration of a simple one step Euler approximation for the ODE (2.10) which has also been studied by Delfour [6] and Reber [17] for time varying systems.

Replacing the derivative in $(2.10)$ by a difference quotient with step size $h / N$, we get the difference equation

$$
z_{k+1}^{N}=\left(I+\frac{h}{N} A^{N}\right) z_{k}^{N}, \quad k \geqq 0,
$$

in $\mathbb{R}^{n(N+1)}$. Since

$$
I+\frac{h}{N} A^{N}=\left[\begin{array}{cccc}
I+\frac{h}{N} A_{0}^{N} & \frac{h}{N} A_{1}^{N} & \cdots & \frac{h}{N} A_{N}^{N} \\
I & 0 & & \\
& \ddots & \ddots & \\
& & I & 0
\end{array}\right],
$$

the $n(N+1)$-dimensional first order difference equation $(2.15)$ is equivalent to the $n$-dimensional $(N+1)$ st order difference equation

$$
\frac{N}{h}\left(x_{k+1}^{N}-x_{k}^{N}\right)=\sum_{j=0}^{N} A_{j}^{N} x_{k-j}^{N}, \quad k \geqq 0,
$$

by means of the identification

$$
z_{k}^{N}=\left(\begin{array}{c}
x_{k}^{N} \\
\vdots \\
x_{k-N}^{N}
\end{array}\right) \in \mathbb{R}^{n(N+1)}
$$


Equation (2.17) may be interpreted as a direct application of a 1-step difference approach to the RFDE (2.1) with $x_{k}^{N}$ approximating $x(k h / N)$. Finally, note that this simplification of the difference equation (2.15) is only possible because of the coincidence of the step size $h / N$ for the time-discretization with the mesh size of the spatial discretization in the subspace $X^{N} \subset M^{2}$.

3. The structure of the approximating systems. It is the goal of this section to analyse in detail the structure of the approximating systems (2.10), (2.14) and (2.15) respectively $(2.17)$. It is shown that there is a strict analogy to the structure of the underlying RFDE (2.1) as it has been described in $\$ 2.2$. In particular, there are certain structural matrices $F^{N}$ and $G^{N}$ playing the same role for the approximating systems as the operators $F$ and $G$ do for the RFDE (2.1).

3.1. The structural matrices. Starting from (2.17), we observe that there is another way of transforming this $(N+1)$ st order difference equation into an equivalent first order equation. For this sake let us rewrite (2.17) as

$$
\begin{aligned}
& \frac{N}{h}\left(x_{k+1}^{N}-x_{k}^{N}\right)=\sum_{j=0}^{k} A_{j}^{N} x_{k-j}^{N}+f_{k+1}^{N}, \quad k \geqq 0, \\
& x_{0}^{N}=f_{0}^{N},
\end{aligned}
$$

where $A_{j}^{N}:=0, f_{j}^{N}:=0$ for $j>N$ and

$$
\begin{aligned}
f_{0}^{N} & =x_{0}^{N}, \\
f_{k}^{N} & =\sum_{j=k}^{N} A_{j}^{N} x_{k-1-j}^{N}, \quad k=1, \cdots, N .
\end{aligned}
$$

The forcing term

$$
f^{N}=\left(\begin{array}{c}
f_{0}^{N} \\
\vdots \\
f_{N}^{N}
\end{array}\right) \in \mathbb{R}^{n(N+1)}
$$

may be considered as the initial state of (3.1) since it contains all the information which is needed for determining the future behavior of its solution $x_{k}^{N}, k \geqq 0$. Correspondingly the state at instant $k \in \mathbb{N}$ is given by $w_{k}^{N} \in \mathbb{R}^{n(N+1)}$ where

$$
\begin{aligned}
& w_{k, 0}^{N}=x_{k}^{N}, \\
& w_{k, l}^{N}=\sum_{j=l}^{k+l-1} A_{j}^{N} x_{k+l-1-j}^{N}+f_{k+l}^{N}, \quad l=1, \cdots, N .
\end{aligned}
$$

Then it is easy to see that $w_{k}^{N}$ satisfies the first order difference equation

$$
w_{k+1}^{N}=\left(I+\frac{h}{N}\left(A_{T}^{N}\right)^{*}\right) w_{k}^{N}, \quad k \geqq 0,
$$

since

$$
I+\frac{h}{N}\left(A_{T}^{N}\right)^{*}=\left[\begin{array}{cccc}
I+\frac{h}{N} A_{0}^{N} & \frac{h}{N} I & & \\
A_{1}^{N} & 0 & I \cdot & \\
\vdots & & \ddots & I \\
A_{N}^{N} & & & 0
\end{array}\right] .
$$

Note that (3.4) can be regarded as a one step Euler approximation for the ODE (2.14). 
We conclude that there are two state concepts for the difference equation (2.17), namely (2.18) and (3.2-3), both of which lead to a first order difference equation in $\mathbb{R}^{n(N+1)}$, namely to $(2.15)$ and (3.4). The relation between these two state concepts can be described by certain structural matrices $F^{N}$ and $G^{N}$. Before defining these matrices, we introduce the concept of a fundamental solution for equation (3.1).

Definition 3.1. The fundamental matrix of equation (3.1) is the sequence $X_{k}^{N} \in$ $\mathbb{R}^{n \times n}, k \geqq 0$, defined by

$$
\frac{N}{h}\left(X_{k+1}^{N}-X_{k}^{N}\right)=\sum_{j=0}^{k} A_{j}^{N} X_{k-j}^{N}, \quad k \in \mathbb{N}, \quad X_{0}^{N}=I .
$$

Remark 3.2. (i) By induction, it is easy to see that

$$
\frac{N}{h}\left(X_{k+1}^{N}-X_{k}^{N}\right)=\sum_{j=0}^{k} X_{k-j}^{N} A_{j}^{N}, \quad k \in \mathbb{N} .
$$

(ii) The solution of (3.1) is given by

$$
x_{k}^{N}=X_{k}^{N} f_{0}^{N}+\frac{h}{N} \sum_{j=0}^{k-1} X_{j}^{N} f_{k-j}^{N}, \quad k \geqq 0 .
$$

Now we introduce the matrices

and

$$
F^{N}=\left[\begin{array}{cccccc}
I & 0 & \cdots & & \cdots & 0 \\
0 & A_{1}^{N} & & & A_{N}^{N} \\
& & & . & \cdot & 0 \\
& & \cdot & . & . & \vdots \\
& & & . & . & \vdots \\
0 & A_{N}^{N} & 0 & & \cdots & 0
\end{array}\right]
$$

$$
G^{N}=K^{N} Q^{N}, \quad K^{N}=\left[\begin{array}{lllc}
X_{N}^{N} & \cdots & \cdots & X_{0}^{N} \\
\vdots & & . \cdot & 0 \\
\vdots & & . \cdot \cdot & \vdots \\
X_{0}^{N} & 0 & \cdots & 0
\end{array}\right] .
$$

Then it is easy to see that $f^{N}=F^{N} z_{0}^{N}$ if $z_{0}^{N} \in \mathbb{R}^{n(N+1)}$ is defined by (2.18) and $f^{N} \in \mathbb{R}^{n(N+1)}$ is the forcing term of (3.1) defined by (3.2). Moreover, if $x_{k}^{N}, k \geqq 0$, is the solution of (3.1) and $z_{N}^{N} \in \mathbb{R}^{n(N+1)}$ is defined by (2.18), then it follows from Remark 3.2 that $z_{N}^{N}=G^{N} f^{N}$. Making use of these facts, one can easily establish the following result which is strictly analogous to Theorem 2.1.

Proposition 3.3.

$$
\left(I+\frac{h}{N} A^{N}\right)^{N}=G^{N} F^{N}, \quad\left(I+\frac{h}{N}\left(A_{T}^{N}\right)^{*}\right)^{N}=F^{N} G^{N} .
$$

$$
F^{N} A^{N}=\left(A_{T}^{N}\right)^{*} F^{N}, \quad A^{N} G^{N}=G^{N}\left(A_{T}^{N}\right)^{*} .
$$

$$
F^{N} e^{A^{N} t}=e^{\left(A_{T}^{N}\right)^{*} t} F^{N}, \quad e^{A^{N} t} G^{N}=G^{N} e^{\left(A_{T}^{N}\right)^{*} t}, \quad t \geqq 0 .
$$


(iv)

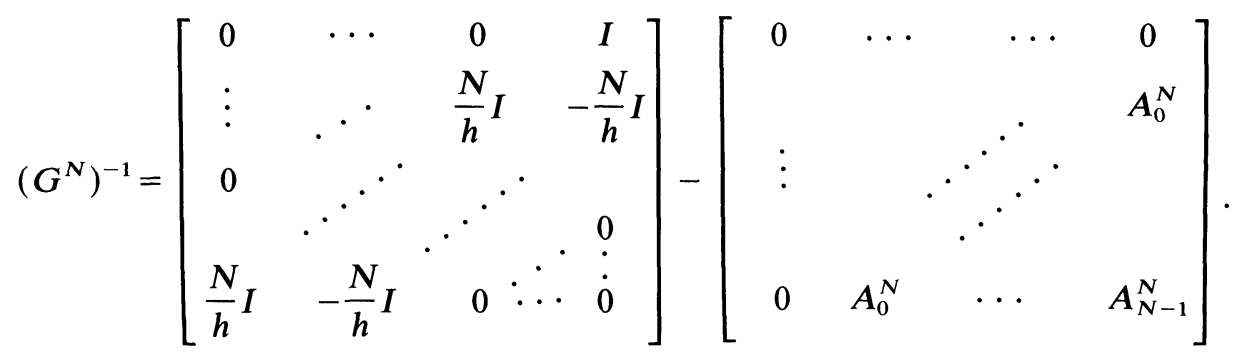

Proof. Let $x_{k}^{N} \in \mathbb{R}^{n}, k \geqq-N$, be a solution of (2.17), let $z_{k}^{N} \in \mathbb{R}^{n(N+1)}, k \geqq 0$, be defined by (2.18) and $f^{N} \in \mathbb{R}^{n(N+1)}$ by (3.2). Then $f^{N}=F^{N} z_{0}^{N}$ and $z_{k}^{N}$ satisfies (2.15) for $k \geqq 0$. Furthermore $x_{k}^{N}, k \geqq 0$, satisfies (3.1) and therefore $z_{N}^{N}=G^{N} f^{N}$. This implies

$$
\left(I+\frac{h}{N} A^{N}\right)^{N} z_{0}^{N}=z_{N}^{N}=G^{N} f^{N}=G^{N} F^{N} z_{0}^{N} .
$$

Thus we have proved the first equation in statement (i). Now let $w_{k}^{N} \in \mathbb{R}^{n(N+1)}, k \geqq 0$, be defined by (3.3). Then $w_{0}^{N}=f^{N}$ and it follows from (3.2) that $w_{k}^{N}=F^{N} z_{k}^{N}$ for every $k \geqq 0$. Since $w_{k}^{N}$ satisfies (3.4), we conclude that

$$
\left(I+\frac{h}{N}\left(A_{T}^{N}\right)^{*}\right) F^{N} z_{0}^{N}=\left(I+\frac{h}{N}\left(A_{T}^{N}\right)^{*}\right) w_{0}^{N}=w_{1}^{N}=F^{N} z_{1}^{N}=F^{N}\left(I+\frac{h}{N} A^{N}\right) z_{0}^{N} .
$$

This proves the first equation in statement (ii).

In order to establish the second equations in (i) and (ii), let $f^{N} \in \mathbb{R}^{n(N+1)}$ be given, let $x_{k}^{N} \in \mathbb{R}^{n}, k \geqq 0$, be the unique solution of (3.1) and let $z_{k}^{N} \in \mathbb{R}^{n(N+1)}, k \geqq N$, and $w_{k}^{N} \in \mathbb{R}^{n(N+1)}, k \geqq 0$, be defined by (2.18) and (3.3), respectively. Then the sequence $x_{k+l}^{N}, l \geqq 0$, satisfies the equation

$$
\frac{N}{h}\left(x_{k+l+1}^{N}-x_{k+l}^{N}\right)=\sum_{j=0}^{l} A_{j}^{N} x_{k+l-j}^{N}+w_{k, l+1}^{N}, \quad l \geqq 0,
$$

and hence it follows from Remark 3.2 (ii) that $z_{k+N}^{N}=G^{N} w_{k}^{N}$ for $k \geqq 0$. Furthermore $w_{0}^{N}=f^{N}$ and $w_{k}^{N}=F^{N} z_{k}^{N}$ for $k \geqq N$. Since $w_{k}^{N}$ satisfies (3.4) for $k \geqq 0$ and $z_{k}^{N}$ satisfies (2.15) for $k \geqq N$, we conclude that

$$
\left(I+\frac{h}{N}\left(A_{T}^{N}\right)^{*}\right)^{N} f^{N}=w_{N}^{N}=F^{N} z_{N}^{N}=F^{N} G^{N} f^{N}
$$

and

$$
G^{N}\left(I+\frac{h}{N}\left(A_{T}^{N}\right)^{*}\right) f^{N}=G^{N} w_{1}^{N}=z_{N+1}^{N}=\left(I+\frac{h}{N} A^{N}\right) z_{N}^{N}=\left(I+\frac{h}{N} A^{N}\right) G^{N} f^{N} .
$$

Thus we have proved the statements (i) and (ii). Statement (iii) is an immediate consequence of (ii).

Finally, let $\left(G^{N}\right)^{-1}$ be defined as in (iv) and let $K^{N}$ be defined by (3.9). Then it follows from (3.5) that $\left(G^{N}\right)^{-1} K^{N}=\left(Q^{N}\right)^{-1}$ and hence $G^{N}=K^{N} Q^{N}$. This proves statement (iv).

Proposition 3.3 shows that, for any solution $z^{N}(t)$ of $(2.10)$, the function $w^{N}(t)=$ $F^{N} z^{N}(t)$ satisfies $(2.14)$ and, conversely, for any solution $w^{N}(t)$ of $(2.14)$, the function $z^{N}(t)=G^{N} w^{N}(t)$ satisfies $(2.10)$. 
3.2. Spectral theory. In this section we give a brief overview over some spectral properties of $A^{N}$ and $\left(A_{T}^{N}\right)^{*}$ which are analogous to well-known results in the theory of RFDEs. In particular, we will see that the rational complex $n \times n$-matrix valued function

$$
\Delta^{N}(\lambda)=\lambda I-L^{N}(\lambda), L^{N}(\lambda)=\sum_{j=0}^{N} A_{j}^{N}\left(\frac{N}{N+\lambda h}\right)^{j}, \quad \lambda \neq-\frac{N}{h},
$$

plays precisely the same role for the approximating systems as the characteristic matrix $\Delta(\lambda)$ does for the underlying RFDE (2.1). Moreover, we introduce the matrices

$$
E_{\lambda}^{N}=\left[\begin{array}{c}
I \\
\frac{N}{N+\lambda h} I \\
\vdots \\
\left(\frac{N}{N+\lambda h}\right)^{N} I
\end{array}\right] \in \mathbb{C}^{n(N+1) \times n}
$$

and

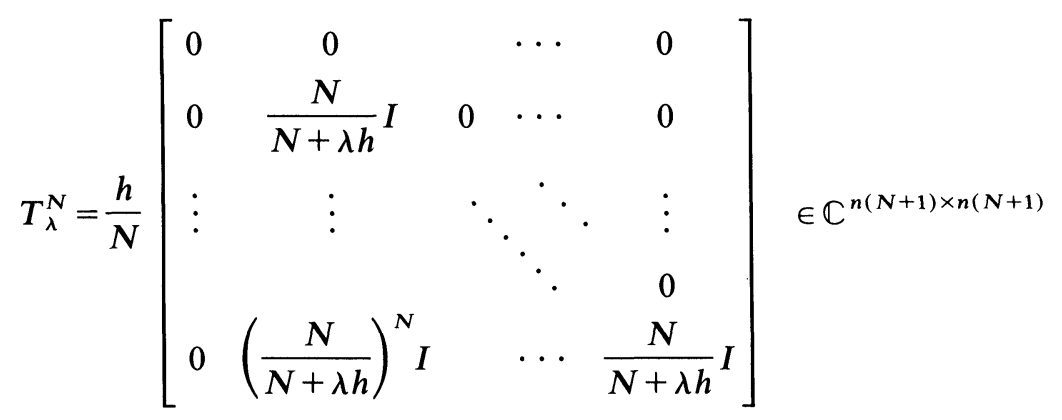

for $\lambda \in \mathbb{C}, \lambda \neq-N / h$.

Lemma 3.4. Let $\lambda \in \mathbb{C}, \lambda \neq-N / h$, and $z, w \in \mathbb{C}^{n(N+1)}$ be given. Then $\left(\lambda I-A^{N}\right) z=w$ if and only if

$$
z=E_{\lambda}^{N} z_{0}+T_{\lambda}^{N} w
$$

and

$$
\Delta^{N}(\lambda) z_{0}=\left(E_{\lambda}^{N}\right)^{T} Q^{N} F^{N} w .
$$

Proof. Clearly $\left(\lambda I-A^{N}\right) z=w$ if and only if $\left(\lambda Q^{N}-H^{N}\right) z=Q^{N} w$ or equivalently

$$
\begin{gathered}
\lambda z_{0}-\sum_{j=0}^{N} A_{j}^{N} z_{j}=w_{0}, \\
z_{j}=\frac{N}{N+\lambda h}\left[\frac{h}{N} w_{j}+z_{j-1}\right], \quad j=1, \cdots, N .
\end{gathered}
$$

Equation (3.16) is equivalent to

$$
z_{j}=\left(\frac{N}{N+\lambda h}\right)^{j} z_{0}+\frac{h}{N} \sum_{\nu=1}^{j}\left(\frac{N}{N+\lambda h}\right)^{\nu} w_{j+1-\nu}, \quad j=1, \cdots, N,
$$


and hence to (3.13). If this is satisfied, then (3.15) is equivalent to

$$
\begin{aligned}
\Delta^{N}(\lambda) z_{0} & =\lambda z_{0}-A_{0}^{N} z_{0}-\sum_{j=1}^{N} A_{j}^{N}\left[z_{j}-\frac{h}{N} \sum_{\nu=1}^{j}\left(\frac{N}{N+\lambda h}\right)^{\nu} w_{j+1-\nu}\right] \\
& =w_{0}+\frac{h}{N} \sum_{\nu=1}^{N}\left(\frac{N}{N+\lambda h}\right)^{\nu} \sum_{j=\nu}^{N} A_{j}^{N} w_{j+1-\nu} \\
& =\left(E_{\lambda}^{N}\right)^{T} Q^{N} F^{N} w .
\end{aligned}
$$

Note that the above lemma is strictly analogous to a well-known result in the theory of RFDEs (see e.g. Hale [10], Delfour-Manitius [7]). It has several important consequences which are summarized in the proposition below and can be proved straightforwardly. Statement (i) can be found in Banks-Burns [2]. Statement (iv) is the analogon of Proposition 2.2.

Proposition 3.5.

(i) Let $\lambda \in \mathbb{C}, \lambda \neq-N / h$; then $\lambda \in \sigma\left(A^{N}\right)$ if and only if $\operatorname{det} \Delta^{N}(\lambda)=0$.

(ii) $\lambda=-N / h \in \sigma\left(A^{N}\right)$ if and only if $\operatorname{det} A_{N}^{N}=0$.

(iii) $\sigma\left(\left(A_{T}^{N}\right)^{*}\right)=\sigma\left(A_{T}^{N}\right)=\sigma\left(A^{N}\right)$.

(iv) If $\lambda \neq-N / h$ and $\operatorname{det} \Delta^{N}(\lambda) \neq 0$, then

$$
\begin{aligned}
& \left(\lambda I-A^{N}\right)^{-1}=E_{\lambda}^{N} \Delta^{N}(\lambda)^{-1}\left(E_{\lambda}^{N}\right)^{T} Q^{N} F^{N}+T_{\lambda}^{N}, \\
& \left(\lambda I-\left(A_{T}^{N}\right)^{*}\right)^{-1}=F^{N} E_{\lambda}^{N} \Delta^{N}(\lambda)^{-1}\left(E_{\lambda}^{N}\right)^{T} Q^{N}+\left(T_{\lambda}^{N}\right)^{T} .
\end{aligned}
$$

Remark 3.6. A solution $x(t)$ of the RFDE (2.1) is said to be small if it vanishes after some finite time $T$ (Henry [11]). If $L: \mathscr{C} \rightarrow \mathbb{R}^{n}$ is given by (2.2) and if $A_{01}(\cdot) \equiv 0$, then there exist nonzero small solutions of (2.1) if and only if $\operatorname{det} A_{q}=0$ (Manitius [14]). Now note that for sufficiently large $N$ this means that $\operatorname{det} A_{N}^{N}=0$ and hence $-N / h \in \sigma\left(A^{N}\right)$ (Proposition 3.5 (ii)). This indicates that the generalized eigenmodes of (2.10) respectively (2.14) corresponding to the eigenvalue $\lambda=-N / h$ play the role of the small solutions in the approximating systems. Moreover, note that the solutions of the difference equation (2.15) starting with generalized eigenvectors of $A^{N}$ corresponding to $\lambda=-N / h$ are precisely those solutions which vanish after a finite time.

4. Convergence and stability. Having introduced a number of operators for the approximating systems which are analogous to well-known operators in the theory of RFDEs, we may pose the question, if-and in what sense-these operators converge. This problem will be considered in the next section.

4.1. Convergence. We begin with some preliminary facts.

Remark 4.1. (i) It is easy to see that the function $\eta^{N}: \mathbb{R} \rightarrow \mathbb{R}^{n \times n}$ defined by

$$
\eta^{N}(\tau)=\lim _{\sigma \uparrow-k h / N} \eta(\sigma), \quad-\frac{k+1}{N} h<\tau \leqq-\frac{k h}{N}, \quad k \in \mathbb{Z},
$$

satisfies the inequality

$$
\int_{-h}^{0}\left|\eta(\tau)-\eta^{N}(\tau)\right| d \tau \leqq \frac{h}{N} \operatorname{VAR}(\eta)
$$

(ii) For every $\lambda \in \mathbb{C}, \lambda \neq-N / h$, let us define the function $e_{\lambda}^{N}:[-h, 0] \rightarrow \mathbb{C}$ by

$$
e_{\lambda}^{N}(\tau)=\left(\frac{N}{N+\lambda h}\right)^{j}, \quad-\frac{j}{N} h \leqq \tau<-\frac{j-1}{N} h, \quad j=0, \cdots, N .
$$


Then it is well known that the limit

$$
\lim _{N \rightarrow \infty} \sup _{-h \leqq \tau \leqq 0}\left|e^{\lambda \tau}-e_{\lambda}^{N}(\tau)\right|=0
$$

exists uniformly on bounded subsets of the complex plane.

The following convergence result for $\Delta^{N}(\lambda)$ has been shown by Banks and Burns [2]. For completeness, we present an alternative and simplified proof.

LEMMA 4.2. (i) $\Delta^{N}(\lambda)$ converges to $\Delta(\lambda)$ uniformly on every bounded subset of the complex plane.

(ii) For every $\alpha \geqq 0$ there exists a constant $c_{\alpha}>0$ such that $\left|L^{N}(\lambda)\right| \leqq c_{\alpha}$ for every $N \in \mathbb{N}$ with $N>\alpha$ h and every $\lambda \in \mathbb{C}$ with $\operatorname{Re} \lambda \geqq-\alpha$.

Proof. Note that

$$
L\left(e^{\lambda \cdot}\right)=\int_{-h}^{0} e^{\lambda \tau} d \eta(\tau)=-\eta(-h) e^{-\lambda h}-\lambda \int_{-h}^{0} \eta(\tau) e^{\lambda \tau} d \tau
$$

and, by (3.10) and (2.12),

$$
\begin{aligned}
L^{N}(\lambda) & =\sum_{j=0}^{N}\left[\eta^{N}\left(-\frac{j-1}{N} h\right)-\eta^{N}\left(-\frac{j}{N} h\right)\right]\left(\frac{N}{N+\lambda h}\right)^{j} \\
& =-\eta^{N}(-h)\left(\frac{N}{N+\lambda h}\right)^{N}-\lambda \frac{h}{N} \sum_{j=1}^{N} \eta^{N}\left(-\frac{j-1}{N} h\right)\left(\frac{N}{N+\lambda h}\right)^{j} \\
& =-\eta(-h) e_{\lambda}^{N}(-h)-\lambda \int_{-h}^{0} \eta^{N}(\tau) e_{\lambda}^{N}(\tau) d \tau .
\end{aligned}
$$

Thus statement (i) follows immediately from Remark 4.1. Statement (ii) follows from (3.10) with $c_{\alpha}=\operatorname{VAR}(\eta) \sup \left\{(N /(N-\alpha h))^{N} \mid N>\alpha h\right\}<\infty$.

For the next result we need the space

$$
M^{\infty}=\mathbb{R}^{n} \times L^{\infty}\left[-h, 0 ; \mathbb{C}^{n}\right]
$$

endowed with the norm $\|\phi\|_{M^{\infty}=\max }\left\{\left|\phi^{0}\right|,\left\|\phi^{1}\right\|_{L^{\infty}}\right\}$ for $\phi \in M^{\infty}$.

THEOREM 4.3. The limits

$$
\lim _{N \rightarrow \infty}\left\|E_{\lambda}-\iota^{N} E_{\lambda}^{N}\right\|_{\mathscr{L}\left(\mathbb{C}^{n}, M^{\infty}\right)}=0=\lim _{N \rightarrow \infty}\left\|T_{\lambda}-\iota^{N} T_{\lambda}^{N} \pi^{N}\right\|_{\mathscr{L}\left(M^{2}, M^{\infty}\right)}
$$

exist uniformly on bounded subsets of the complex plane.

Proof. The statement on $E_{\lambda}$ is an immediate consequence of Remark 4.1 (ii), since $\iota^{N} E_{\lambda}^{N} x=\left(x, e_{\lambda}^{N}(\cdot) x\right) \in M^{\infty}$ for $x \in \mathbb{C}^{\mathrm{n}}, N \in \mathbb{N}$ and $\lambda \in \mathbb{C}, \lambda \neq-N / h$.

In order to prove the second part of the theorem, let us first define $e(\lambda, \tau) \in M^{2}$ by

$$
e(\lambda, \tau)^{0}=0, \quad e(\lambda, \tau)^{1}(\sigma)= \begin{cases}0, & -h \leqq \sigma<\tau, \\ e^{\bar{\lambda}(\tau-\sigma)}, & \tau \leqq \sigma \leqq 0,\end{cases}
$$

for $\lambda \in \mathbb{C}$ and $-h \leqq \tau \leqq 0$. Then

$$
\left[T_{\lambda} p^{N} \phi\right]^{1}(\tau)=\left\langle e(\lambda, \tau), p^{N} \phi\right\rangle=\left\langle p^{N} e(\lambda, \tau), \phi\right\rangle .
$$

for all $\phi \in M^{2}, \lambda \in \mathbb{C}, N \in \mathbb{N}$ and $\tau \in[-h, 0]$. Moreover, the closure of the set $\{e(\lambda, \tau)|| \lambda \mid \leqq c,-h \leqq \tau \leqq 0\}$ in $M^{2}$ is compact and thus $p^{N}$ converges uniformly on this set. Hence $T_{\lambda} p^{N}$ converges to $T_{\lambda}$ in $\mathscr{L}\left(M^{2}, M^{\infty}\right)$ uniformly on bounded subsets of the complex plane.

Secondly, note that

$$
\left[\iota^{N} T_{\lambda}^{N} z\right]^{1}(\tau)=\frac{h}{N} \sum_{\nu=1}^{j}\left(\frac{N}{N+\lambda h}\right)^{\nu} z_{j+1-\nu}=\int_{-j h / N}^{0} e_{\lambda}^{N}(\sigma)\left[\iota^{N} z\right]^{1}\left(-\frac{j h}{N}-\sigma\right) d \sigma
$$


and hence

$$
\begin{aligned}
& \left|\left[T_{\lambda} p^{N} \phi\right]^{1}(\tau)-\left[\iota^{N} T_{\lambda}^{N} \pi^{N} \phi\right]^{1}(\tau)\right| \\
& \leqq \\
& \quad\left|\left[T_{\lambda} p^{N} \phi\right]^{1}(\tau)-\left[T_{\lambda} p^{N} \phi\right]^{1}\left(-\frac{j h}{N}\right)\right| \\
& \quad+\int_{-j h / N}^{0}\left|e^{\lambda \sigma}-e_{\lambda}^{N}(\sigma)\right|\left|\left[p^{N} \phi\right]^{1}\left(-\frac{j h}{N}-\sigma\right)\right| d \sigma
\end{aligned}
$$

for $-j h / N \leqq \tau<-(j-1) h / N, j=1, \cdots, N$. Thus the statement of the theorem follows from Remark 4.1 (ii) together with the fact that the set

$$
\left\{\left[T_{\lambda} \phi\right]^{1}|| \lambda \mid \leqq c, \phi \in M^{2},\|\phi\|<1\right\}
$$

is equicontinuous for $c<\infty$.

THEOREM 4.4. For every $\phi \in M^{2}$

$$
F \phi=\lim _{N \rightarrow \infty} \iota^{N} F^{N} \pi^{N} \phi .
$$

Proof. We prove this result in 3 steps. The first step is a formula for the operators $\pi^{N} F$ and $F^{N} \pi^{N}$.

Step 1. $\left[\pi^{N} F \phi\right]_{0}=\left[F^{N} \pi^{N} \phi\right]_{0}=\phi^{0}$ and for $j=1, \cdots, N$

$$
\begin{gathered}
{\left[\pi^{N} F \phi\right]_{j}=-\frac{N}{h} \int_{-h}^{h / N} \eta\left(\tau-\frac{j}{N} h\right)\left[\phi^{1}(\tau)-\phi^{1}(\tau-h / N)\right] d \tau,} \\
{\left[F^{N} \pi^{N} \phi\right]_{j}=-\frac{N}{h} \int_{-h}^{h / N} \eta^{N}\left(\tau-\frac{j}{N} h\right)\left[\phi^{1}(\tau)-\phi^{1}(\tau-h / N)\right] d \tau .}
\end{gathered}
$$

Proof. Let us define $\phi^{1}(\tau):=0$ for $t \notin[-h, 0]$. Then

$$
\begin{aligned}
{\left[\pi^{N} F \phi\right]_{j}=} & \frac{N}{h} \int_{-j h / N}^{-(j-1) h / N} \int_{-h}^{0} d \eta(\tau) \phi^{1}(\tau-\sigma) d \sigma \\
= & \frac{N}{h} \int_{-h}^{0} d \eta(\tau) \int_{\tau+(j-1) h / N}^{\tau+j h / N} \phi^{1}(\sigma) d \sigma \\
= & -\frac{N}{h} \eta(-h) \int_{-h+(j-1) h / N}^{-h+j h / N} \phi^{1}(\sigma) d \sigma \\
& -\frac{N}{h} \int_{-h}^{0} \eta(\tau)\left[\phi^{1}\left(\tau+\frac{j}{N} h\right)-\phi^{1}\left(\tau+\frac{j-1}{N} h\right)\right] d \tau \\
= & -\frac{N}{h} \eta(-h) \int_{-h}^{-h+j h / N}\left[\phi^{1}(\tau)-\phi^{1}\left(\tau-\frac{h}{N}\right)\right] d \tau \\
& -\frac{N}{h} \int_{-h+j h / N}^{h / N} \eta\left(\tau-\frac{j}{N} h\right)\left[\phi^{1}(\tau)-\phi^{1}\left(\tau-\frac{h}{N}\right)\right] d \tau \\
= & -\frac{N}{h} \int_{-h}^{h / N} \eta\left(\tau-\frac{j}{N} h\right)\left[\phi^{1}(\tau)-\phi^{1}\left(\tau-\frac{h}{N}\right)\right] d \tau
\end{aligned}
$$


since $\eta(\tau)=\eta(-h)$ for $\tau \leqq-h$, and

$$
\begin{aligned}
{\left[F^{N} \pi^{N} \phi\right]_{j} } & =\sum_{\nu=j}^{N} A_{\nu}^{N}\left[\pi^{N} \phi\right]_{\nu-j+1} \\
& =\frac{N}{h} \sum_{\nu=j}^{N} \int_{-(\nu-j+1) h / N}^{-(\nu-j) h / N}\left[\eta^{N}\left(\tau-\frac{j-1}{N} h\right)-\eta^{N}\left(\tau-\frac{j}{N} h\right)\right] \phi^{1}(\tau) d \tau \\
& =\frac{N}{h} \int_{-h}^{0}\left[\eta^{N}\left(\tau-\frac{j-1}{N} h\right)-\eta^{N}\left(\tau-\frac{j}{N} h\right)\right] \phi^{1}(\tau) d \tau \\
& =-\frac{N}{h} \int_{-h}^{h / N} \eta^{N}\left(\tau-\frac{j}{N} h\right)\left[\phi^{1}(\tau)-\phi^{1}\left(\tau-\frac{h}{N}\right)\right] d \tau
\end{aligned}
$$

for $j=1, \cdots, N$. In the last but one equation we have used the fact that

$$
\eta^{N}\left(\tau-\frac{j-1}{N} h\right)=\eta^{N}\left(\tau-\frac{j}{\bar{N}} h\right) \text { for } \tau \leqq-\frac{N-j+1}{N} h
$$

This proves step 1 .

Step 2. $\left\|F^{N}\right\|_{N} \leqq \max \{1, \operatorname{VAR}(\eta)\}, \forall N \in \mathbb{N}$.

Proof. By the well-known convolution inequality, we have

$$
\begin{aligned}
\sum_{j=1}^{N}\left|\left[F^{N} z\right]_{j}\right|^{2} & =\sum_{j=1}^{N}\left|\sum_{\nu=j}^{N} A_{\nu}^{N} z_{\nu-j+1}\right|^{2} \\
& \leqq\left[\sum_{\nu=1}^{N}\left|A_{\nu}^{N}\right|\right]^{2} \sum_{j=1}^{N}\left|z_{j}\right|^{2} \leqq[\operatorname{VAR}(\eta)]^{2} \sum_{j=1}^{N}\left|z_{j}\right|^{2}
\end{aligned}
$$

and hence

$$
\begin{aligned}
\left\|F^{N} z\right\|_{N}^{2} & =\left|z_{0}\right|^{2}+\frac{h}{N} \sum_{j=1}^{N}\left|\left[F^{N} z\right]_{j}\right|^{2} \\
& \leqq\left|z_{0}\right|^{2}+[\operatorname{VAR}(\eta)]^{2} \frac{h}{N} \sum_{j=1}^{N}\left|z_{j}\right|^{2} \leqq[\max \{1, \operatorname{VAR}(\eta)\}]^{2}\|z\|_{N}^{2}
\end{aligned}
$$

for $z \in \mathbb{R}^{n(N+1)}$ and $N \in \mathbb{N}$. This proves step 2 .

Step 3. $F \phi=\lim _{N \rightarrow \infty} \iota^{N} F^{N} \pi^{N} \phi, \forall \phi \in M^{2}$.

Proof. Let us first assume that $\phi^{1}$ is continuously differentiable and that $\phi^{1}(0)=0$. Moreover let us define $\phi^{1}(\tau)=0$ for $\tau>0$. Then it follows from step 1 and (4.1) that

$$
\begin{aligned}
& \left|\left[\pi^{N} F \phi\right]_{j}-\left[F^{N} \pi^{N} \phi\right]_{j}\right| \\
& \quad=\left|\int_{-h}^{h / N}\left[\eta^{N}\left(\tau-\frac{j h}{N}\right)-\eta\left(\tau-\frac{j h}{N}\right)\right] \frac{N}{h}\left[\phi^{1}(\tau)-\phi^{1}(\tau-h / N)\right] d \tau\right| \\
& \quad \leqq \int_{-h}^{0}\left|\eta^{N}(\tau)-\eta(\tau)\right| d \tau \cdot \sup _{-h+h / N \leqq \tau \leqq h / N} \frac{N}{h}\left|\int_{\tau-h / N}^{\tau} \dot{\phi}^{1}(\sigma) d \sigma\right| \\
& \quad \leqq \frac{h}{N} \operatorname{VAR}(\eta)\left\|\dot{\phi}^{1}\right\|_{\infty}
\end{aligned}
$$


and hence

$$
\begin{aligned}
\left\|F \phi-\iota^{N} F^{N} \pi^{N} \phi\right\| & \leqq\left\|F \phi-p^{N} F \phi\right\|+\left\|\pi^{N} F \phi-F^{N} \pi^{N} \phi\right\| \\
& \leqq\left\|F \phi-p^{N} F \phi\right\|+\frac{h}{N}\left[\sum_{j=1}^{N}\left|\left[\pi^{N} F \phi\right]_{j}-\left[F^{N} \pi^{N} \phi\right]_{j}\right|^{2}\right]^{1 / 2} \\
& \leqq\left\|F \phi-p^{N} F \phi\right\|+\frac{h^{2}}{N^{3 / 2}} \operatorname{VAR}(\eta)\left\|\dot{\phi}^{1}\right\|_{\infty}
\end{aligned}
$$

For any $\phi \in M^{2}$ the statement follows from the Banach-Steinhaus theorem and step 2. This proves the theorem.

Combining the above convergence results with the concrete representation of the resolvent operators given in Proposition 2.2 and Proposition 3.5, we obtain the following result. The proof is a straightforward application of Lemma 6.1 and will be omitted.

Corollary 4.5. The limits

$$
\begin{aligned}
& \lim _{N \rightarrow \infty}\left\|(\lambda I-A)^{-1}-\iota^{N}\left(\lambda I-A^{N}\right)^{-1} \pi^{N}\right\|_{\mathscr{L}\left(M^{2}, M^{\infty}\right)}=0, \\
& \lim _{N \rightarrow \infty}\left\|\left(\lambda I-A_{T}^{*}\right)^{-1}-\iota^{N}\left(\lambda I-\left(A_{T}^{N}\right)^{*}\right)^{-1} \pi^{N}\right\|_{\mathscr{L}\left(M^{2}\right)}=0
\end{aligned}
$$

exist uniformly on those bounded subsets of the complex plane which are uniformly bounded away from the zeros of $\operatorname{det} \Delta(\lambda)$.

THEOREM 4.6.

$$
\lim _{N \rightarrow \infty}\left\|G-\iota^{N} G^{N} \pi^{N}\right\|_{\mathscr{L}\left(M^{2}, M^{\infty}\right)}=0
$$

Proof. We establish this result in three steps.

Step 1. Let $X_{j}^{N}, j \geqq 0$, be given by (3.5) and let us define

$$
X^{N}(t):=X_{j}^{N}, \quad \frac{j}{N} h \leqq t<\frac{j+1}{N} h, \quad j=0,1,2, \cdots
$$

Then $X^{N}(t)$ converges to $X(t)$ uniformly on every compact interval $[0, T]$.

Proof. For every $k \in \mathbb{N}$

$$
\begin{aligned}
X_{k}^{N} & =I+\sum_{\nu=0}^{k-1}\left[X_{\nu+1}^{N}-X_{\nu}^{N}\right] \\
& =I+\frac{h}{N} \sum_{\nu=0}^{k-1} \sum_{j=0}^{\nu} A_{\nu-j}^{N} X_{j}^{N} \\
& =I+\frac{h}{N} \sum_{j=0}^{k-1} \sum_{\nu=j}^{k-1} A_{\nu-j}^{N} X_{j}^{N} \\
& =I-\frac{h}{N} \sum_{j=0}^{k-1} \eta^{N}\left(\frac{j+1-k}{N} h\right) X_{j}^{N} \\
& =I-\int_{0}^{k h / N} \eta^{N}\left(s-\frac{k h}{N}\right) X^{N}(s) d s
\end{aligned}
$$


and hence for $k h / N \leqq t<(k+1) h / N$

$$
\begin{aligned}
X(t)-X^{N}(t)= & X(t)-X\left(\frac{k h}{N}\right) \\
& +\int_{0}^{k h / N}\left[\eta^{N}\left(s-\frac{k h}{N}\right)-\eta\left(s-\frac{k h}{N}\right)\right] X(s) d s \\
& +\int_{0}^{k h / N} \eta^{N}\left(s-\frac{k h}{N}\right)\left[X^{N}(s)-X(s)\right] d s
\end{aligned}
$$

Thus the desired convergence result follows from Gronwall's lemma.

Step 2. Let $z \in \mathbb{R}^{n(N+1)}$ and $j \in\{1, \cdots, N\}$. Then

$$
\begin{aligned}
{\left[\iota^{N} G^{N} z\right]^{0} } & =X^{N}(h) z_{0}+\int_{0}^{h} X^{N}(s)\left[\iota^{N} z\right]^{1}(s-h) d s \\
{\left[\iota^{N} G^{N} z\right]^{1}(\tau) } & =X^{N}(h+\tau) z_{0}+\int_{0}^{h} X^{N}(s)\left[\iota^{N} z\right]^{1}\left(s-h+\frac{j h}{N}\right) d s, \\
& -\frac{j}{N} h \leqq \tau<-\frac{j-1}{N} h .
\end{aligned}
$$

Proof. If $-j h / N \leqq \tau<-(j-1) h / N$, then

$$
\begin{aligned}
{\left[\iota^{N} G^{N} z\right]^{1}(\tau) } & =X_{N-j}^{N} z_{0}+\frac{h}{N} \sum_{l=0}^{N-j-1} X_{l}^{N} z_{N-j-l} \\
& =X^{N}(h+\tau) z_{0}+\sum_{l=0}^{N-j-1} \int_{l h / N}^{(l+1) h / N} X^{N}(s)\left[\iota^{N} z\right]^{1}\left(s-h+\frac{j h}{N}\right) d s \\
& =X^{N}(h+\tau) z_{0}+\int_{0}^{h} X^{N}(s)\left[\iota^{N} z\right]^{1}\left(s-h+\frac{j h}{N}\right) d s .
\end{aligned}
$$

In the case $j=0$ this equation leads to the desired expression for $\left[\iota^{N} G^{N} x\right]^{0}$.

Step 3. $\lim _{N \rightarrow \infty}\left\|G-\iota^{N} G^{N} \pi^{N}\right\|_{\left(M^{2}, M^{\infty}\right)}=0$.

Proof. First note that the functions $[G f]^{1} \in \mathscr{C}, f \in M^{2},\|f\| \leqq 1$, are equicontinuous since the canonical embedding of $W^{1,2}$ into $\mathscr{C}$ is a compact operator.

Now let $z \in \mathbb{R}^{n(N+1)}$. Then, by step 2 ,

$$
\left[G \iota^{N} z-\iota^{N} G^{N} z\right]^{0}=\left[X(h)-X^{N}(h)\right] z_{0}+\int_{0}^{h}\left[X(s)-X^{N}(s)\right]\left[\iota^{N} z\right]^{1}(s-h) d s
$$

and for $-j h / N \leqq \tau<-(j-1) h / N, j=1, \cdots, N$,

$$
\begin{aligned}
{\left[G \iota^{N} z-\iota^{N} G^{N} z\right]^{1}(\tau)=} & {\left[G \iota^{N} z\right]^{1}(\tau)-\left[G^{N} z\right]^{1}\left(-\frac{j h}{N}\right)+\left[X\left(h-\frac{j h}{N}\right)-X^{N}\left(h-\frac{j h}{\bar{N}}\right)\right] z_{0} } \\
& +\int_{0}^{h}\left[X(s)-X^{N}(s)\right]\left[\iota^{N} z\right]^{1}\left(s+\frac{j h}{N}-h\right) d s .
\end{aligned}
$$

By step 1 and the equicontinuity mentioned above, this implies

$$
\lim _{N \rightarrow \infty}\left\|G^{N}-\iota^{N} G^{N}\right\|_{\mathscr{L}\left(\mathbb{R}^{n(N+1)}, M^{\infty}\right)}=0 .
$$

Moreover, note that the operator $G: M^{2} \rightarrow M^{\infty}$ is compact. So is the extended adjoint 
operator $G^{*}:\left(M^{\infty}\right)^{*} \rightarrow M^{2}$. By Lemma 6.1, this implies

$$
\lim _{N \rightarrow \infty}\left\|G-G p^{N}\right\|_{\mathscr{L}\left(M^{2}, M^{\infty}\right)}=\lim _{N \rightarrow \infty}\left\|G^{*}-p^{N} G^{*}\right\|_{\mathscr{L}\left(\left(M^{\infty}\right)^{*}, M^{2}\right)}=0 .
$$

Hence the statement of the theorem follows from the inequality

$$
\left\|G-\iota^{N} G^{N} \pi^{N}\right\|_{\mathscr{L}\left(M^{2}, M^{\infty}\right)} \leqq\left\|G-G p^{N}\right\|_{\mathscr{L}\left(M^{2}, M^{\infty}\right)}+\left\|G \iota^{N}-\iota^{N} G^{N}\right\|_{\mathscr{L}\left(\mathbb{R}^{n(N+1)}, M^{\infty}\right)} .
$$

Let $f \in M^{2}$ be given and let $x(t), t \geqq 0$, be the corresponding solution of (2.5). Moreover, let $x^{N}(t), t \geqq 0$, be defined by

$$
x^{N}(t)=x_{k}^{N}, \quad \frac{k}{N} h \leqq t<\frac{k+1}{N} h, \quad k \geqq 0,
$$

where $x_{k}^{N}, k \geqq 0$, is the unique solution of (3.1) corresponding to $f^{N}=\pi^{N} f \in \mathbb{R}^{n(N+1)}$. Then the previous theorem shows that

$$
\lim _{N \rightarrow \infty} \sup _{[0, T]}\left|x(t)-x^{N}(t)\right|=0
$$

and moreover that this convergence is uniform for bounded $f \in M^{2}$. This has also been proved by Reber [17, Thm. 7.5] under the condition that $L: \mathscr{C} \rightarrow \mathbb{R}^{n}$ is given by (2.2).

Let us now introduce the operator families $S^{N}(t) \in \mathscr{L}\left(M^{2}\right), S_{T}^{N^{*}}(t) \in \mathscr{L}\left(M^{2}\right), t \geqq 0$, by

$$
\begin{aligned}
& S^{N}(t)=\iota^{N}\left[I+\frac{h}{N} A^{N}\right]^{k} \pi^{N}, \quad S_{T}^{N^{*}}(t)=\iota^{N}\left[I+\frac{h}{N}\left(A_{T}^{N}\right)^{*}\right]^{k} \pi^{N}, \\
& \frac{k}{N} h \leqq t<\frac{k+1}{N} h, \quad k=0,1,2, \cdots .
\end{aligned}
$$

Then the following result is a direct consequence of Theorem 4.4 and Theorem 4.6 together with the factorization results (Theorem 2.1 (i) and Proposition 3.3 (i)).

Corollary 4.7. (i) For all $\phi \in M^{2}, f \in M^{2}$

$$
S(t) \phi=\lim _{N \rightarrow \infty} S^{N}(t) \phi, \quad S_{T}^{*}(t) f=\lim _{N \rightarrow \infty} S_{T}^{N^{*}}(\tau) f
$$

and the convergence is uniform on every compact interval $[0, T]$.

(ii) For every $k \in \mathbb{N}$

$$
\lim _{N \rightarrow \infty}\left\|S(k h)-S^{N}(k h)\right\|_{\mathscr{L}\left(M^{2}, M^{\infty}\right)}=\lim _{N \rightarrow \infty}\left\|S_{T}^{*}(k h)-S_{T}^{N^{*}}(k h)\right\|_{\mathscr{L}\left(M^{2}\right)}=0 .
$$

Proof. It only remains to note-for the proof of statement (ii) - that, by Lemma 6.1,

$$
\begin{aligned}
\lim _{N \rightarrow \infty}\left\|S(h)-G \iota^{N} F^{N} \pi^{N}\right\|_{\mathscr{L}\left(M^{2}, M^{\infty}\right)} & \\
& =\lim _{N \rightarrow \infty}\left\|F^{*} G^{*}-\iota^{N}\left(F^{N}\right)^{T} \pi^{N} G^{*}\right\|_{\mathscr{L}\left(\left(M^{\infty}\right)^{*}, M^{2}\right)}=0 .
\end{aligned}
$$

Statement (ii) of the above result is apparently new. The strong convergence of statement (i) has been stated without proof by Delfour [6]. The strong convergence of $S^{N}(t)$ has been shown by Reber [17] and Rosen [19].

4.2. Uniform stability. It is a simple consequence of Corollary 4.7 that the discrete time systems (2.15) and (3.4) are stable in a uniform sense if the underlying RFDE (2.1) is exponentially stable. More precisely, we have the following result.

THEOREM 4.8. Let $\omega<0$ and suppose that $\operatorname{det} \Delta(\lambda) \neq 0$ for every $\lambda \in \mathbb{C}$ with $\operatorname{Re} \lambda \geqq \omega$. Then there exist an $N_{0} \in \mathbb{N}$ and a constant $\gamma>0$ such that for every $N \geqq N_{0}$

$$
\left\|\left(I+\frac{h}{N} A^{N}\right)^{k}\right\|_{N} \leqq \gamma e^{\omega k h / N} .
$$


Proof. It follows from a well-known result in semigroup theory that there exists a $k_{0} \in \mathbb{N}$ such that $\left\|S\left(k_{0} h\right)\right\|_{\mathscr{L}\left(M^{2}\right)}<e^{\omega k_{0} h}$. By Corollary 4.7 (ii), this implies the existence of an $N_{0} \in \mathbb{N}$ such that

$$
\left\|\left(I+\frac{h}{N} A^{N}\right)^{k_{0} N}\right\|_{N}<e^{\omega k_{0} N}, \quad N \geqq N_{0} .
$$

Moreover, it follows from Corollary 4.7 (i), that

$$
\gamma:=e^{-\omega k_{0} h} \sup \left\{\left\|\left(I+\frac{h}{N} A^{N}\right)^{l}\right\|_{N} \mid l=0, \cdots, k_{0} N-1, N \in N\right\}<\infty .
$$

We conclude that the following inequality holds for $N \geqq N_{0}$ and $k=\nu k_{0} N+l$ with $\nu \in \mathbb{N}$ and $l \in\left\{0, \cdots, k_{0} N-1\right\}$

$$
\begin{aligned}
\left\|\left(I+\frac{h}{N} A^{N}\right)^{k}\right\|_{N} & \leqq\left(I+\frac{h}{N} A^{N}\right)^{l}\left\|_{N}\right\|\left(I+\frac{h}{N} A^{N}\right)^{k_{0} N} \|_{N}^{\nu} \\
& \leqq \gamma e^{\omega k_{0} h} e^{\omega \nu k_{0} h} \leqq \gamma e^{\omega\left(\nu k_{0}+l / N\right) h}=\gamma e^{\omega k h / N} .
\end{aligned}
$$

It follows easily from Lemma 4.2 that the stability of the RFDE (2.1) also implies the stability of the approximating continuous-time systems (2.10) and (2.14) if $N$ is sufficiently large (the precise arguments are given in the proof of Theorem 4.9 below). However, a uniform estimate in the spirit of Theorem 4.8 has not yet been proved in the literature on these approximation schemes. It has been stated as a conjecture by Gibson [9] and provides-in that paper-a crucial step in the convergence proof for the solutions of the algebraic Riccati equation. Repin [18] also claims the uniform stability of the approximating systems (2.10), however, his arguments are extremely unclear and it seems almost impossible to convert them into a rigorous proof. The following theorem closes this important gap in the approximation theory of RFDE's and may be considered as the main result of this paper.

THEOREM 4.9. Let L: $\mathscr{C} \rightarrow \mathbb{R}^{N}$ be given by (2.2) and let the RFDE (2.1) be exponentially stable. Then the approximating systems (2.10) and (2.14) are uniformly exponentially stable for sufficiently large $N$. This means that there exists an $N_{0} \in \mathbb{N}$ and constants $\varepsilon>0$, $\gamma \geqq 1$ such that

$$
\left\|e^{A^{N}}\right\|_{N},\left\|e^{\left(A_{T}^{N}\right)^{*} t}\right\|_{N} \leqq \gamma e^{-\varepsilon t}
$$

for every $t \geqq 0$ and every $N \geqq N_{0}$.

Proof. First note that the statement on $\left(A_{T}^{N}\right)^{*}$ follows from that on $A^{N}$. Secondly, it follows from Theorem 6.2 and the exponential estimates in Theorem 2.3. (ii) that it is enough to show that there exists an $N_{0} \in \mathbb{N}$ and a constant $c>0$ such that

$$
\int_{0}^{\infty}\left\|e^{A^{N} t} z\right\|_{N}^{2} d t \leqq c^{2}\|z\|_{N}^{2}
$$

for every $z \in \mathbb{R}^{n(N+1)}$ and every $N \geqq N_{0}$. We will prove this in 5 steps.

Step 1. There exists an $N_{0} \in \mathbb{N}$ such that $\operatorname{det} \Delta^{N}(\lambda) \neq 0$ for every $\lambda \in \mathbb{C}$ with $\operatorname{Re} \lambda \geqq 0$ and every $N \geqq N_{0}$.

Proof. By Lemma 4.2.(ii), the complex function det $\Delta^{N}(\lambda)$ cannot have a zero in the closed right halfplane outside the disc of radius $\operatorname{VAR}(\eta)$ centered at the origin. Inside this disc the nonexistence of unstable eigenvalues of $A^{N}$ follows from Lemma 4.2.(i) if $N$ is sufficiently large. 
Step 2. For $N \in \mathbb{N}$ let us introduce the matrix

$$
a^{N}=\frac{N}{h}\left[\begin{array}{rrrr}
-1 & & & \\
1 & \ddots & & \\
& \ddots & \ddots & \\
& & 1 & -1
\end{array}\right] \in \mathbb{R}^{N \times N}
$$

Then there exist constant $\varepsilon_{0}>0, \gamma_{0} \geqq 1$ such that

$$
\left|e^{a^{N} t}\right|_{N \times N} \leqq \gamma_{0} e^{-\varepsilon_{0} t} \quad \forall t \geqq 0, \quad \forall N \in \mathbb{N} .
$$

Proof. First of all it is easy to see that $x^{T} a^{N} x \leqq 0$ for every $x \in \mathbb{R}^{N}$ and every $N \in N$. Hence it follows from a well-known result in semigroup theory that

$$
\left|e^{a^{N}}\right|_{N \times N} \leqq 1 \quad \forall t \geqq 0, \quad \forall N \in \mathbb{N},
$$

where $|\cdot|_{N \times N}$ denotes the operator norm on $\mathbb{R}^{N \times N}$ which corresponds to the Euclidean norm on $\mathbb{R}^{N}$. Moreover

$$
\begin{aligned}
& e^{a^{N} t}=e^{-N t / h}\left[\begin{array}{cccc}
1 & & & \\
\frac{N t / h}{1 !} & \ddots & & \\
\vdots & \ddots & & \\
\frac{(N t / h)^{N-1}}{(N-1) !} & \cdots & \frac{N t / h}{1 !} & 1
\end{array}\right] \\
& e^{-N t / h} \sum_{k=0}^{N-1}\left[\begin{array}{cccccc}
0 & \cdots & \cdots & \cdots & 0 \\
\frac{(N t / h)^{k}}{k !} & & & & \vdots \\
0 & \ddots & \ddots & & \\
\vdots & \ddots & & & \vdots \\
0 & \cdots & 0 & \frac{(N t / h)^{k}}{k !} & 0
\end{array}\right]
\end{aligned}
$$

and hence

$$
\left|e^{a^{N} t}\right|_{N \times N} \leqq \sum_{k=0}^{N-1} \frac{1}{k !}\left(\frac{N t}{h}\right)^{k} e^{-N t / h}
$$

for every $t \geqq 0$ and every $N \in \mathbb{N}$. Since

$$
\int_{0}^{\infty} t^{k} e^{-N t / h} d t=k !(h / N)^{k+1},
$$

this implies

$$
\int_{0}^{\infty}\left|e^{a^{N} t}\right|_{N \times N} d t \leqq \sum_{k=0}^{N-1} \frac{h}{N}=h .
$$

Together with (4.4) this estimate proves the statement of step 2 (Theorem $6.2, p=1$ ). More precisely, $\varepsilon_{0}>0$ may be chosen to be any constant less than $1 / h$.

Step 3. For every $z \in \mathbb{R}^{n(N+1)}$ and every $N \in \mathbb{N}$

$$
\int_{-\infty}^{\infty}\left\|T_{i \omega}^{N} z\right\|_{N}^{2} d \omega \leqq \frac{\pi \gamma_{0}^{2}}{\varepsilon_{0}}\|z\|_{N}^{2} .
$$


Proof. First note that

$$
\left(\lambda I_{N}-a^{N}\right)^{-1}=\frac{h}{N}\left[\begin{array}{ccc}
\frac{N}{N+\lambda h} & & \\
\vdots & \ddots & \\
\left(\frac{N}{N+\lambda h}\right)^{N} & \cdots & \frac{N}{N+\lambda h}
\end{array}\right]
$$

and hence

$$
T_{\lambda}^{N}=\left[\begin{array}{cc}
0 & 0 \\
0 & \left(\lambda I_{N}-a^{N}\right)^{-1} \otimes I_{n}
\end{array}\right]
$$

Now let $z \in \mathbb{R}^{n(N+1)}$ be given. Then, by step 2 , the function

$$
\tilde{z}(t)=\left[e^{a^{N}} \otimes I_{n}\right]\left[\begin{array}{c}
z_{1} \\
\vdots \\
z_{N}
\end{array}\right] \in \mathbb{R}^{n N}, \quad t \geqq 0,
$$

is square integrable on the interval $[0, \infty)$ and its Fourier transform

$$
\hat{z}(i \omega)=\frac{1}{\sqrt{2 \pi}} \int_{0}^{\infty} e^{-i \omega t} \tilde{z}(t) d t=\frac{1}{\sqrt{2 \pi}}\left[\left(i \omega I_{N}-a^{N}\right)^{-1} \otimes I_{n}\right] \tilde{z}(0)
$$

satisfies $\|\hat{z}\|_{L^{2}\left[-\infty, \infty ; \mathbb{C}^{n N}\right]}=\|\tilde{z}\|_{L^{2}\left[0, \infty ; \mathbb{R}^{n N}\right] \text {. Hence it follows from (4.5) and step } 2 \text { that }}$

$$
\begin{aligned}
\int_{-\infty}^{\infty}\left\|T_{i \omega}^{N} z\right\|_{N}^{2} d \omega & =\frac{h}{N} \int_{-\infty}^{\infty}\left|\left[\left(i \omega I_{N}-a^{N}\right)^{-1} \otimes I_{n}\right] \tilde{z}(0)\right|_{\mathbb{C}^{n N}}^{2} d \omega \\
& =\frac{2 \pi h}{N} \int_{-\infty}^{\infty}|\hat{z}(i \omega)|_{\mathbb{C}^{n N}}^{2} d \omega \\
& =\frac{2 \pi h}{N} \int_{0}^{\infty}|\tilde{z}(t)|_{\mathbb{R}^{n N}}^{2} d t \\
& \leqq \frac{2 \pi h}{N} \int_{0}^{\infty} \gamma_{0}^{2} e^{-2 \varepsilon_{0} t} d t|\tilde{z}(0)|_{\mathbb{R}^{n N}}^{2} \\
& \leqq \frac{\pi \gamma_{0}^{2}}{\varepsilon_{0}}\|z\|_{N}^{2} .
\end{aligned}
$$

This proves step 3 .

Step 4. There exists a constant $c>0$ such that the following inequality holds for every $z \in \mathbb{R}^{n(N+1)}$ and every $N \geqq N_{0}$

$$
\int_{-\infty}^{\infty}\left\|\left(i \omega I-A^{N}\right)^{-1} z\right\|_{N}^{2} d \omega \leqq 2 \pi c^{2}\|z\|_{N}^{2}
$$

Proof. Recall that

$$
\left(i \omega I-A^{N}\right)^{-1}=E_{i \omega}^{N} \Delta^{N}(i \omega)^{-1}\left(E_{i \omega}^{N}\right)^{T} Q^{N} F^{N}+T_{i \omega}^{N}, \quad \omega \in \mathbb{R},
$$

(Proposition 3.5). By step 3, it remains to establish the desired inequality for the first term on the right-hand side of this equation. Moreover, it follows from Theorem 4.4 that the operators $F^{N}$ are uniformly bounded and it is easy to see that the operators 
$E_{\lambda}^{N}$ and $\left(E_{\bar{\lambda}}^{N}\right)^{*}=\left(E_{\lambda}^{N}\right)^{T} Q^{N}$ are uniformly bounded on the imaginary axis. Thus it remains to prove the desired estimate for the term $\Delta^{N}(i \omega)^{-1}$. But for $|\omega|>\operatorname{VAR}(\eta)$ it follows from Lemma 4.2 (ii) that

$$
\left|\Delta^{N}(i \omega)^{-1}\right|=\left|\sum_{k=0}^{\infty}(i \omega)^{-k-1} L^{N}(i \omega)^{k}\right| \leqq \frac{1}{|\omega|-\operatorname{VAR}(\eta)} .
$$

This inequality, together with Lemma 4.2 (i) shows that

$$
\sup _{N \geqq N_{0}} \int_{-\infty}^{\infty}\left|\Delta^{N}(i \omega)^{-1}\right|^{2} d \omega<\infty \text {. }
$$

This proves step 4 .

Step 5. For every $z \in \mathbb{R}^{n(N+1)}$ and every $N \geqq N_{0}$

$$
\int_{0}^{\infty}\left\|e^{A^{N_{t}}} z\right\|_{N}^{2} d t \leqq c^{2}\|z\|_{N}^{2} .
$$

Proof. Let $z \in \mathbb{R}^{n(N+1)}$ and $z(t)=e^{A^{N} t} z$ for $t \geqq 0$ and $N \geqq N_{0}$. Then $z(t)$ is square integrable on $(0, \infty)$ and its Fourier transform is given by $\hat{z}(i \omega)=(2 \pi)^{-1 / 2}\left(i \omega I-A^{N}\right)^{-1}$. By the Fourier-Plancherel theorem and step 4, we obtain

$$
\int_{0}^{\infty}\left\|e^{A^{N} t} z\right\|_{N}^{2} d t=(2 \pi)^{-1} \int_{-\infty}^{\infty}\left\|\left(i \omega I-A^{N}\right)^{-1} z\right\|_{N}^{2} d \omega \leqq c^{2}\|z\|_{N}^{2} .
$$

This proves step 5 and the statement of the theorem.

Remark 4.10. The uniform exponential decay rate $-\varepsilon$ for the approximating systems $(2.10),(2.14)$ which has been found in the proof of Theorem 4.9 is always larger than $-1 / h$. The question remains open if one can find a uniform exponential bound for the approximating systems with the exponential decay rate $\omega_{0}+\varepsilon$ where $\omega_{0}=\sup \{\operatorname{Re} \lambda \mid \operatorname{det} \Delta(\lambda)=0\}$ and $\varepsilon>0$ can be chosen arbitrarily small. It is also an open problem if the operators $\iota^{N} e^{a^{N} t} \pi^{N}$ converge to the (compact) operators $S(t) \in$ $\mathscr{L}\left(M^{2}\right)$ in the uniform operator topology if $t \geqq h$. If this could be shown, then the solution to the uniform stability problem mentioned above would be an immediate consequence.

5. Conclusions. The present paper studies in detail certain finite dimensional approximations for linear retarded systems, namely the averaging approximation scheme, both a continuous and a discrete time version as well as the relation between these two. It turns out that these finite dimensional approximations show-under several aspects-precisely the same structure as the underlying RFDE. In particular, the duality relations are of the same type and there are certain structural operators which play an important role for the description of the approximating systems and are analogous to those which have recently been introduced by Bernier-Manitius [3], Manitius [14], Delfour-Manitius [7] for the study of RFDE's. Moreover, it is shown that these operators actually converge to the corresponding operators in the theory of retarded systems. One of these convergence results, namely Theorem 4.6 , is only a slight extension of a corresponding result by Reber [17, Thm. 7.5].

Based on this detailed analysis of the structure of the approximating systems, it is shown that both the discrete- and the continuous-time approximations are stable in a uniform sense if the underlying RFDE is asymptotically stable. Such a result is by no means obvious and not all approximation schemes have this property. For example, it is shown in Kappel-Salamon [12] that spline approximations for RFDE's can never have the property of uniform stability. Nevertheless, the uniform stability result provides 
a crucial step in the convergence proof of Gibson [9] for the solutions of the algebraic Riccati equation. Moreover, the structural matrix $F^{N}$ introduced in this paper allows a factorization of the approximate Riccati operator in precisely the same manner as it is shown in Kappel-Salamon [12] for the spline approximation scheme. Finally, it seems likely that the uniform stability results of this paper have some implications for the construction of finite dimensional compensators for RFDE's. This is a research problem for future investigations.

6. Appendix. In this section we formulate and prove two general functional analytic results which are needed frequently in $\S 4$.

LEMMA 6.1. Let $A$ be an arbitrary set and let $X, Y, Z$ be Banach spaces. Moreover, let $K^{\alpha} \in \mathscr{L}(X, Y), T^{\alpha} \in \mathscr{L}(Y, Z), T_{k}^{\alpha} \in \mathscr{L}(Y, Z), \alpha \in A, k \in \mathbb{N}$, be bounded, linear operators with the properties

(i) $\operatorname{cl}\left\{K^{\alpha} x \mid \alpha \in A, x \in X,\|x\| \leqq 1\right\} \subset Y$ is compact,

(ii) $T^{\alpha} y=\lim _{k \rightarrow \infty} T_{k}^{\alpha} y$ for all $y \in Y$ uniformly in $\alpha \in A$.

Then $T_{k}^{\alpha} K^{\alpha}$ tends to $T^{\alpha} K^{\alpha} \in \mathscr{L}(X, Z)$ in the uniform operator topology as $k$ tends to infinity and this convergence is uniform in $\alpha \in A$.

Proof. Note that for every $\varepsilon>0$ there exist finitely many $x_{1}, \cdots, x_{n} \in X$ and $\alpha_{1}, \cdots, \alpha_{n} \in A$ such that for every $\alpha \in A$ and every $x \in X$ with $\|x\| \leqq 1$ there is a $j \in\{1, \cdots, n\}$ such that $\left\|K^{\alpha} x-K^{\alpha_{j}} x_{j}\right\| \leqq \varepsilon$. Hence the desired uniform convergence result follows from the inequality

$$
\left\|T_{k}^{\alpha} K^{\alpha} x-T^{\alpha} K^{\alpha} x\right\| \leqq\left[\left\|T_{k}^{\alpha}\right\|+\left\|T^{\alpha}\right\|\right]\left\|K^{\alpha} x-K^{\alpha_{j}} x_{j}\right\|+\left\|T_{k}^{\alpha} K^{\alpha_{j}} x_{j}-T^{\alpha} K^{\alpha_{j}} x_{j}\right\| .
$$

The next result is a quantitative estimate for the equivalence of $L^{p}$-stability and exponential stability for strongly continuous semigroups. This equivalence has been proved-for the case $p=2$-by several authors, see for example Datko [5], CurtainPritchard [4], Przyluski [16]. But none of these give the desired quantitative estimate which is essential for the proof of uniform stability in Theorem 4.9. Again in the case $p=2$ such a quantitative estimate can be found without proof in Gibson [8]. We mention that some of the ideas in the proof of the theorem below are taken from Przyluski [16, Prop. 9] and Zabczyk [21, Thm. 5.1].

THEOREM 6.2. Let $S(t), t \geqq 0$, be a strongly continuous semigroup of bounded, linear operators on a Banach space $X$ satisfying the exponential bound

$$
\|S(t)\|_{\mathscr{L}(X)} \leqq M e^{\omega t}, \quad t \geqq 0,
$$

for some constants $M \geqq 1, \omega \geqq 0$. Moreover, let $1 \leqq p<\infty$ and suppose that there exists a constant $c>0$ such that

$$
\int_{0}^{\infty}\|S(t) x\|^{p} d t \leqq c^{p}\|x\|^{p}, \quad x \in X .
$$

Then, for every

$$
\alpha>-\frac{1}{p c^{p} M^{p}},
$$

there exists a $\gamma=\gamma(\alpha, \omega, M, c, p) \geqq 1$ such that

$$
\|S(t)\|_{\mathscr{L}(X)} \leqq \gamma e^{\alpha t}, \quad t \geqq 0 .
$$

Remark 6.3. If $-1 / p c^{p} M^{p}<\alpha<0$, then there exists a unique $T>0$ satisfying

$$
e^{-\alpha p T}=1+\frac{T}{c^{p} M^{p} e^{\omega p T}}
$$


or equivalently

$$
\alpha=\frac{1}{p T} \log \frac{c^{p} M^{p} e^{\omega p T}}{T+c^{p} M^{p} e^{\omega p T}} .
$$

The proof of Theorem 6.2 shows that in this case $\gamma=\gamma(\alpha, \omega, M, c, p) \geqq 1$ can be chosen as

$$
\gamma=\frac{\left[T+c^{p} M^{p} e^{\omega p T}\right]^{2 / p}}{T^{1 / p} c} .
$$

Proof of Theorem 6.2. Let $T>0$ be given and let us define

$$
\varepsilon=\varepsilon(T)=\frac{T}{T+c^{p} M^{p} e^{\omega p T}}>0 .
$$

Then it follows from (6.1) and (6.2) that

$$
\begin{aligned}
\sum_{k=0}^{\infty}\left\|S(T)^{k} x\right\|^{p} & =\sum_{k=0}^{\infty} \frac{1}{T} \int_{0}^{T}\|S(k T) x\|^{p} d t \\
& \leqq\|x\|^{p}+\sum_{k=1}^{\infty} \frac{1}{T} \int_{0}^{T}\|S(T-t)\|^{p}\|S((k-1) T+t) x\|^{p} d t \\
& \leqq\|x\|^{p}+\frac{1}{T}\left[\sup _{[0, T]}\|S(t)\|^{p}\right] \int_{0}^{\infty}\|S(t) x\|^{p} d t \\
& \leqq\left[1+\frac{c^{p} M^{p} e^{\omega p T}}{T}\right]\|x\|^{p} \\
& =\varepsilon^{-1}\|x\|^{p},
\end{aligned}
$$

for every $x \in X$. This implies that

$$
\sum_{k=0}^{\infty}\left\|S(T)^{k+1} x\right\|^{p}=\sum_{k=0}^{\infty}\left\|S(T)^{k} x\right\|^{p}-\|x\|^{p} \leqq(1-\varepsilon) \sum_{k=0}^{\infty}\left\|S(T)^{k} x\right\|^{p}
$$

and hence

$$
\begin{aligned}
\left\|S(T)^{m} x\right\| & \leqq\left[\sum_{k=0}^{\infty}\left\|S(T)^{k+m} x\right\|^{p}\right]^{1 / p} \\
& \leqq(1-\varepsilon)^{m / p}\left[\sum_{k=0}^{\infty}\left\|S(T)^{k} x\right\|^{p}\right]^{1 / p} \\
& \leqq(1-\varepsilon)^{m / p} \varepsilon^{-1 / p}\|x\|
\end{aligned}
$$

for every $x \in X$ and every $m \in \mathbb{N}$. Now let $t=m T+\tau \geqq 0$ with $m \in \mathbb{N}$ and $0 \leqq \tau<T$. Then we conclude that

$$
\begin{aligned}
\|S(t) x\| & \leqq\|S(\tau)\|\left\|S(T)^{m} x\right\| \\
& \leqq M e^{\omega T} \varepsilon^{-1 / p}(1-\varepsilon)^{m / p}\|x\| \\
& \leqq M e^{\omega T} \varepsilon^{-1 / p} \exp \left(\frac{m}{p} \log (1-\varepsilon)\right) \exp \left(\frac{\log (1-\varepsilon)}{p}\left(\frac{\tau}{T}-1\right)\right)\|x\| \\
& =M e^{\omega T} \varepsilon^{-1 / p}(1-\varepsilon)^{-1 / p} \exp \left(\frac{\log (1-\varepsilon)}{p T}(m T+\tau)\right)\|x\| \\
& =\gamma e^{\alpha t}\|x\|
\end{aligned}
$$


where

$$
\alpha=\alpha(T)=\frac{1}{p T} \log \frac{c^{p} M^{p} e^{\omega p T}}{T+c^{p} M^{p} e^{\omega p T}}<0
$$

and

$$
\gamma=\gamma(T)=M e^{\omega T}\left[\frac{1}{\varepsilon(1-\varepsilon)}\right]^{1 / p}=\frac{\left[T+c^{p} M^{p} e^{\omega p T}\right]^{2 / p}}{T^{1 / p} c}
$$

(compare (6.5) and (6.6)). Thus the statement of the theorem follows from the fact that $\alpha(T)$ is strictly increasing for $T>0$ and satisfies

$$
\lim _{T \rightarrow 0} \alpha(T)=-\frac{1}{p c^{p} M^{p}}
$$

\section{REFERENCES}

[1] H. T. BANKS AND J. A. BURNS, An abstract framework for approximate solutions to optimal control problems governed by hereditary system, Proc. International Conference on Differential Equations, H. A. Antosiewicz, ed., Academic Press, New York, 1975, pp. 10-25.

[2] - Hereditary control problems: numerical methods based on averaging approximation, this Journal, 16 (1978), pp. 169-208.

[3] C. BERNIER AND A. MANITIUS, On semigroups in $\mathbb{R}^{n} \times L^{p}$ corresponding to differential equations with delays, Canad. J. Math., 30 (1978), pp. 897-914.

[4] R. F. Curtain And A. J. Pritchard, Infinite Dimensional Linear Systems Theory, Lecture Notes in Control and Information Science 8, Springer-Verlag, Berlin, 1978.

[5] R. DATKO, Uniform asymptotic stability of evolutionary processes in Banach space, SIAM J. Math. Anal., 3 (1972), pp. 428-554.

[6] M. C. DELFOUR, The linear quadratic optimal control problem for hereditary differential systems: theory and numerical solution, Appl. Math. Optim., 3 (1977), pp. 101-162.

[7] M. C. Delfour AND A. MANitius, The structural operator F and its role in the theory of retarded systems, Part I, J. Math. Anal. Appl., 73 (1980), pp. 466-490; Part II, J. Math. Anal. Appl., 74 (1980), pp. 359-381.

[8] J. S. Gibson, The Riccati integral equations for optimal control problems in Hilbert space, this Journal, 17 (1979), pp. 537-565.

[9] - Linear quadratic optimal control of hereditary differential systems: infinite dimensional Riccati equations and numerical approximations, this Journal, 21 (1983), pp. 95-139.

[10] J. K. HALE, Theory of Functional Differential Equations, Springer-Verlag, New York, 1977.

[11] D. HENRY, Small solutions of linear autonomous functional differential equations, J. Differential Equations, 8 (1970), pp. 494-501.

[12] F. KAPPEL AND D. SAlAMON, Spline approximation for retarded systems and the Riccati equation, MRC, Univ. of Wisconsin-Madison, TSR \#2680, 1984.

[13] A. MANITIUS, Controllability, observability, and stabilizability of retarded systems, Proc. 1976 IEEE Conference on Decision and Control, IEEE Publications, New York, 1976, pp. 752-758.

[14] Completeness and F-completeness of eigenfunctions associated with retarded functional differential equations, J. Differential Equations, 35 (1980), pp. 1-29.

[15] R. K. Miller, Linear Volterra integro-differential equations as semigroups, Funkcial. Ekvac., 17 (1974), pp. 629-634.

[16] K. M. PRZYLUSKI, The Lyapunov equation and the problem of stability for linear bounded discrete-time systems in Hilbert space, Appl. Math. Optim., 6 (1980), pp. 97-112.

[17] D. D. REBER, A finite difference technique for solving optimization problems governed by linear functional differential equations, J. Differential Equations, 32 (1979), pp. 193-232.

[18] YU. M. REPIN, On the approximate replacement of systems with lag by ordinary differential equations, J. Appl. Math. Mech., 29 (1966), pp. 254-264.

[19] G. I. Rosen, A discrete approximation framework for hereditary systems, J. Differential Equations, 40 (1981), pp. 377-449.

[20] D. Salamon, Control and Observation of Neutral Systems, RNM 91, Pitman, London, 1984.

[21] J. ZABCZYK, Remarks on the control of discrete time distributed parameter systems, this Journal, 12 (1974), pp. 721-735. 